\title{
Response to and perception of nurses during the COVID-19: a qualitative assessment
}

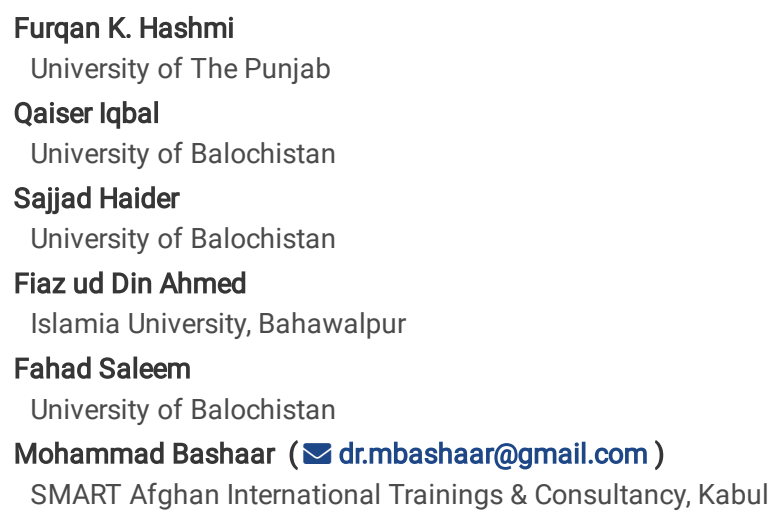




\section{Abstract}

\section{Background}

The COVID-19 outbreak was declared pandemic by the WHO on 11 March 2020. For that reason, we gathered insight from frontline healthcare professionals (nurses) regarding their perceptions, awareness, and familiarity of COVID-19.

\section{Methods}

Being a novel outbreak, we opted for a qualitative research design. With the help of a self-constructed and validated interview guide, face-to-face interviews were conducted with nurses to gain an in-depth knowledge. All interviews were audio-taped, transcribed verbatim, and were then analyzed for thematic contents by the standard content analysis framework.

\section{Results}

The saturation was reached at the $12^{\text {th }}$ interview. Two more interviews were carried out to ensure the saturation achieved at the $12^{\text {th }}$ interview. Thematic content analysis yielded four major themes. (1) Perceived knowledge, awareness, and severity of COVID-19, (2) perceived personal capability of handling and managing the COVID-19, (3) perceived organizational capability of handling and managing the COVID-19, and (4) barriers in handling and managing the COVID-19.

\section{Conclusion}

Findings of our study and reservations of our nurses' highlight areas of concern and scheme of improvement in the effective management of the COVID-19. The respondents are faced with substantial difficulties and barriers in providing healthcare services to patients. We believe that the current study can help in planning policies and generating guidelines that can meet the healthcare needs of the society while managing the COVID-19 as well as for future pandemic outbreaks of the same nature.

\section{Introduction}

Among public health emergencies, an infectious disease outbreak is one of the most imminent threats. Infectious diseases are a leading cause of mortality and the biggest disablers of human functions [1]. Pathogenic microorganisms spread diseases resulting in minor ailments to the pandemic crises [2]. Within this context, zoonotic diseases or zoonoses are infectious diseases of animals that cause infection when transmitted to humans. Around the globe, an estimated $60 \%$ of all infectious diseases are zoonotic and have resulted in millions of deaths in recent years [3]. Furthermore, 30 new human pathogens were identified in the past three decades out of which $75 \%$ originated from animals [4]. The increasing trend in the emergence and transmission of zoonotic diseases is a major health catastrophe and needs both short and long term strategies to minimize disease-related complications, injuries and sufferings.

While discussing zoonotic diseases, humans are yet again faced by a disease that became pandemic in a limited time. The Novel Corona or Coronavirus disease 2019 (COVID-19) was first detected in China that spread to 90 international locations within no time [5]. Initially originated from bats (betacoronavirus) and caused by the "SARS-CoV-2" virus, the exact clinical reasoning related to COVID-19 in humans is yet to be established. Reporting from mild symptoms to severe, including illness resulting in death, the COVID-19 resulted in serious illness among 16\% of all reported cases [5]. As expected, immunocompromised patients and patients with chronic disorders of heart and lung are at higher risk of developing serious COVID-19 illness. Nevertheless, this is just the beginning and ongoing investigations are in process. Till today ( $7^{\text {th }}$ April 2020), 1,214,973 positive cases were reported in 203 countries (territories) and have resulted in 67,841 deaths because of COVID-19 [6]. As the disease is progressing, it is for sure that more cases of COVID-19 will be reported in the coming days and sustained person-to-person spread will also continue to occur. Unfortunately, until today there are no approved medications for the treatment. Therefore, nonpharmaceutical interventions and empirical treatment remain the most important response strategy [6].

The COVID-19 is recognized as a global crisis and efforts around the globe are focused concomitantly on limiting the transmission and reducing the impact of the virus [7]. The outbreak of COVID-19 is a public health emergency as there are fear, anxiety, and panic around the globe. Additionally, the emergence of COVID-19 has resulted in health-related stigma that has created an additional concern. This stigma is generating an atmosphere of uncertainty and chaos in the society [8]. In normal conditions, with known prognosis, treatment and management of a disease, it is relatively easier for healthcare professionals to handle an emergency. However, with a disease like COVID-19 where less is known, healthcare professionals themselves are faced with multiple challenges and are developing stigma about the disease and the patients suffering from the diseases $[9,10]$.

While addressing a pandemic condition, healthcare's capacity to respond to emergencies plays a key role in disease management. At the same time, the awareness, readiness, and preparedness of frontline healthcare professionals are also essential in tackling disease related complications and transmission. Within this context, among all healthcare professionals, nurses play a pivotal role as they are at the frontline of patient care [11, 12]. Furthermore, nurses' role in disease surveillance and infection control is of paramount importance in disease management [1]. However, when it comes to infectious diseases, nurses' awareness of established epidemics has been understood in an unclear manner [1]. Although the literature does report nurses' perceptions and experience of their roles in epidemic response $[13,14]$, information on nurses' preparedness of infectious disease outbreak management is scarce. As the COVID-19 is a recent pandemic, it is imperative to explore not only the clinical appearance of the disease but also the perceptions and knowledge of healthcare professionals (nurses) who work in risky and chaotic environments. Moreover, as there are more myths than evidence-based information circulating the globe, evaluation of nurses' insight of COVID-19 becomes indispensable. For that reason, we gathered insight from nurses practicing in a public hospital of Quetta city regarding their perceptions, awareness, and familiarity of COVID-19. We are sure that the results of this study will contribute to identifying nurses' knowledge gaps in 
response to COVID-19 handling and management. Consequently, the current study explored nurses' knowledge, reflections, and perceptions towards the COVID-19.

\section{Methods}

\section{Study design}

We adopted a qualitative study design (face-to-face interviews) for manifold reasons. This method is flexible and consents to an in-depth exploration of respondents' attitudes, experiences, and intentions $[15,16]$. In addition, qualitative studies generate a wide range of ideas and opinions that individuals carry about issues, as well as divulge viewpoint and differences among groups $[17,18]$. Furthermore, for under-discovered research areas, qualitative methods fill the gaps that are left unexposed by survey-based research [19]. Therefore, inline to the objectives of this study, a qualitative design was a superlative choice for inductive approaches aimed at generating concepts and hypothesis which have far more potential for research than any other models [20].

\section{Study settings, participants and sampling}

The study (interviews) was conducted at Sandeman Provincial Hospital (SPH), Quetta. Established in 1939 and located in the center of the city, SPH is a tertiary care, teaching institute.

Additionally, SPH is normally the institute of choice for the majority of the residents [21]. Registered nurses practicing at the hospital and consenting to participate in the study were approached for face-to-face interviews. We opted for the snowball sampling method [22] and the data were collected until saturation was reached and no new findings were drawn [23].

\section{The interview guide (development, validation, and reliability)}

By keeping in view the information provided by World Health Organization and Centres for Disease Control and Prevention, the research team constructed a semi-structured interview guide $[5,6]$. The guide was later reviewed and validated by a group of experienced physicians and infectious disease specialists. The guide was constructed in the English language and was translated into Urdu (National language of Pakistan) by an independent linguistic expert. The translated guide was back-translated into English to avoid discrepancies by another independent translator [24]. The amended guide was piloted with four nurses to ensure that topics to be discussed were at the level that respondents would comprehend with ease. The preliminary data and conclusion confirmed that the discussion topics were sufficient and appropriately phrased to answer research questions and to minimize validity and reliability threats. As the validity and reliability of the discussion guide were ensured, it was made available for the main study. Data and participants of the pilot study were not included in the final analysis.

\section{Interview procedure and data analysis}

The third author was involved in conducting the interviews. All participants were briefed about the study before the interviews. A debriefing session was again conducted at the end of the discussion. The interviews started with an ice-breaking session. Probing questions were asked in between conversations to clarify the meanings of responses and to gain insight of the topic being discussed.

Each interview lasted for approximately half an hour. The interviews were audio-recorded and were subjected to transcribed verbatim [25]. To draw in-depth views, the freedom to express additional reviews and comments was given to respond. Besides, field notes were also used to complement the recordings. Keeping the nature of the study and the ease of the respondents into consideration, the interviews were conducted in Urdu. The research team analyzed all transcripts and re-analysis of the themes and contents were confirmed. An informal gathering was arranged after the data analysis was completed. The nurses were presented with the final analysis and asked for confirmation of precision and accuracy of words, ideas, and jargon used during the data analysis process. Finally, the transcripts were translated back into English by another independent translator.

\section{Results}

\section{Demographic characteristics of the study respondents}

Although the saturation was reached at the $12^{\text {th }}$ interview, we carried out two more interviews to completely assure the dissemination of findings. Hence fourteen nurses were interviewed. Ten nurses belonged to the age group of $28-47$ years. A majority $(8,57.2 \%)$ had BSc in nursing and $43.0 \%$ had an experience of $11-15$ years (Table 1 ).

Table 1: Demographic characteristics of study respondents $(n=14)$ 


\begin{tabular}{|l|c|c|}
\hline Characteristics & Frequency (N) & Percentage (\%) \\
\hline Age group & 4 & \\
28-37 & 10 & 28.5 \\
38-47 & 2 & 71.5 \\
\hline Current position & 14.3 \\
Matron & 3 & 21.4 \\
Head Nurse & 9 & 64.3 \\
Staff Nurse & & \\
\hline Highest qualification & 8 & 57.2 \\
BSc nursing & 6 & 42.8 \\
BSN & 4 & 28.5 \\
\hline Job experience (years) & & 28.5 \\
0-5 & 4 & 43.0 \\
6-10 & 6 & \\
\hline
\end{tabular}

During the analysis, four major themes were identified and are discussed as under:

\section{Theme 1: Perceived knowledge, awareness, and severity of COVID-19}

During the interviews, nurses were asked about their current knowledge, awareness, and severity of COVID-19. All of the respondents were well responsive to the disease dynamics and the severity level. The Ministry of Health was recognized as the main source of information [26]. Additionally, as the COVID-19 became pandemic is a limited period, nurses by them started to learn about the condition so it can be communicated among their peers and public. The nurses also reported that the information regarding COVID-19 was disseminated in a disciplined manner and hence little trouble was faced regarding evidencebased information selection when it came to COVID-19.

"The Novel Corona or the Corona disease is viral in nature. Disease origin and transmission dynamics are also frightening. This is a serious condition and needs remedial action on an urgent basis." $(N, 2)$

"A deadly viral infection originating from Wuhan, China that became pandemic in no time. It is a critical situation for the world." $(N, 11)$

Additionally, four of the participants also stated that in addition to the information provided by the Ministry of Health, they are in touch with online resources (WHO, CDC, UN, etc) to get a better insight into the disease and the current statistics.

"The disease is new to the world. Being a healthcare practitioner, we must get maximum information about the disease so we can manage the patients to avoid transmission and contamination. We (nurses) need to be vigilant as ignorance can cause irreparable loss to the people and society." ( $N$, 10)

However, two nurses claimed that the environment is overloaded with information. Therefore, at times it is hard to accept or reject the characteristics and statistics related to the disease.

"At times I am confused; there is information everywhere. Everyone (people, print, mass, and social media) is talking about Corona. There is information overload and at times it becomes a problem that what information is right and what is wrong." $(N, 4)$

\section{Theme 2: Perceived personal capability of handling and managing the COVID-19}

The respondents were asked about their capabilities of handling and managing the disease. Mixed results were obtained for this assessment. A handful of the nurses were confident that they are ready to tackle the issues related to COVID-19. Furthermore, they were also positive that as the policymakers are seriously working to counter the disease, they are motivated and ready to respond to the COVID-19.

"At present, the management of COVID-19 is a procedural matter that requires both precautionary methods and treatment of symptoms. With the help of the Ministry of Health, I am well aware of the procedures and positive at the same time that we can manage the disease professionally." ( $N$, 1)

On the other hand, certain reservations regarding the management of COVID-19 were reported. In a general context, nurses believed that the disease is manageable under certain conditions if they are provided with the necessary equipment and facilities at their practicing site.

"We are well aware of the management protocol. The issue is about the continuous availability and accessibility of diagnostic and safety kits at the practicing site. With these materials in hand, we are ready to tackle the disease." $(N, 3)$

"We (nurses) are professionals and we are always ready to face and handle a crisis. For me, it is not about a question of personal capability. You can be the best at your work, but to handle diseases like COVID-19, we need an aggressive approach with facilities and protective gear. Without these, I am sorry; the situation will deteriorate at an alarming rate." $(N, 5)$

\section{Theme 3: Perceived organizational capability of handling and managing the COVID-19}

The thematic analysis reported several issues while talking about organizational capabilities of handling and managing the COVID-19. Nurses had a range of opinions and showed strong reluctance while responding to this query. Likewise, almost all respondents mentioned poor availability of protective materials (kits, masks, gloves, etc) at the practicing site thus making it very difficult for them to manage the disease. 
"The hospital in normal days operates in order. However, during this crisis, we are facing a shortage of masks, gloves, sanitizers, and diagnostic kits. It is impossible to continue our services as the institute itself is facing a severe shortage of materials." $(N, 7)$

Lack of workforce and deprived allocation of space was also mentioned by the respondents. It was also observed that as the hospital is already overloaded, the need for specialist physicians and nurses (infectious diseases) is imminent and should be provided to the hospital on an emergency basis.

"We are a developing nation. We are faced with limited budgets and a lack of specialized healthcare. Dealing with COVID-19 needs the experience of ID physicians and nurses and these specialists should be appointed or transfer as soon as possible to our institute." $(N, 2)$

"This hospital is already overloaded. We will be unable to handle an emergency if the disease spreads to the society. We need strict measures, as like China. The authorities should immediately come up with a space designated only for COVID-19 patients and suspects." (N, 9)

The respondents also mentioned that the Ministry is trying its level best in providing facilities to the healthcare system. Maximum efforts are in progress and at this time of crisis, it is their moral obligation to work in limited supplies and resources.

"The Ministry is serious in her approach. With the limited budget, it is still striving hard to provide facilities and materials to healthcare. Let us join hands and work in a limited environment so that a mass crisis is avoided." $(N, 10)$

"Yes we have issues; every country fighting with COVID-19 is facing the same. It is high time not to brag out deficiencies and work in collaboration so that we can handle this disease effectively and efficiently." $(N, 1)$

\section{Theme 4: Barriers in handling and managing the COVID-19}

Several barriers in handling and managing the COVID-19 were identified. Societal myths and religious stigma were mentioned as a potential barrier to the management of COVID-19. Relating to this observation, where certain myths are circulating in the society, stigma, and taboos are shaping as a hindrance in managing the disease. This is vital to address as people are following instructions that have nothing to do with the disease and the actual precautionary practices are avoided.

"People are recommending onion as a preventive measure against COVID-19. Some are also favoring use of ginger and garlic. I have also heard people buying and using ascorbic acid (vitamin C). This may be beneficial but the actual precautionary practices (hand wash, sneezing methods, social distancing, etc) are not in general discussion." ( $N, 11)$

"As sufferings and death come from Almighty and it is irreversible, people are least serious in taking preventive measures. But the Almighty has also instructed to opt for preventive measures. Although religious leaders are pushing hard to convey this message, the religious stigma is still prevalent and must be addressed on a priority basis." $(N, 8)$

Additionally, panic related to COVID-19 was also reported as a barrier in handling and managing the COVID-19. As the COVID-19 started to become pandemic, the panic resulted in a shortage of masks, gloves and hand sanitizers all over Pakistan including Quetta city [27]. With unavailability of the protective materials, it is getting hard to manage and handle the patients or suspects with COVID-19.

"The disease has created a panic in the society. People have blindly purchased masks, gloves, and sanitizers from pharmacies. The smuggling of masks and gloves was also in the news. Because of this, healthcare professionals are facing a shortage in the hospitals and a barrier in providing care to patients." $(N, 9)$

Another major barrier reported was the movement of suspects from cross borders. The province of Baluchistan shares its open borders with Afghanistan and Iran [28]. Pilgrims visiting Iran and Iraq from all over Pakistan travel through Quetta city. The COVID-19 being endemic in Iran places Quetta city at high risk as the pilgrims are a close suspect of carrying the virus [29].

"With a huge border, it is vital to closely monitor people entering the province of Baluchistan. Unfortunately, the monitoring started very late and there are reports that many suspects entered the province without clinical assessment and diagnosis. For me, this poor monitoring and entry of suspects is the main barrier. We have to stop the influx from the border so that we can concentrate at one point." $(N, 11)$

\section{Discussion}

To the best of our knowledge, the present paper is the first qualitative assessment of nurses' views of the COVID-19. Nurses are the most trusted professionals and one of the biggest segments of the workforce in healthcare [30]. They play a vital role in the delivery of healthcare services and the administration of treatment protocols that is imperative for essential patient care [31]. In today's COVID-19 crisis, it is important to know about the emergency awareness, preparedness and, barriers faced by frontline nurses so that optimal measures and steps can be taken before a mass disaster. Additionally, the limitations and challenges faced by the nurses and highlighted in this paper will also help the policymakers to respond to their queries and to provide enough facilities so that nurses can deal with the COVID-19 disease without fear of being harmed.

The findings suggest that nurses are well aware and prepared to handle the disease as well as a crisis if occurs. However, they are also faced with certain reservations and limitations at the same time. In light of what is reported by the nurses of the current study, we will be discussing each theme separately to get a better insight. 


\section{Knowledge, awareness, and severity of COVID-19}

When dealing with a pandemic situation like COVID-19, responsible and reliable information must be available to the healthcare professionals. A positive finding of the study indicated that our respondents were well aware of the COVID-19 situation. Furthermore, validated and reliable sources were accessed by the respondents to get in-depth information that is an encouraging sign in disease management and treatment. The role played by the Ministry of Health in disseminating reliable information was also appreciated by the study respondents. As credible and quality information is critical for making health-related decisions, our respondents were confident with their current knowledge of COVID-19 that that is an encouraging sign in disease handing and management.

On the other hand, the abundance of COVID-19 related information on the media was mentioned by a few of our respondents hence making it difficult for them to filter accurate situational data and statistics. Although mass media plays an important role in communicating and raising awareness about health research and services, at the time is also generates stories that argue with rational health policies and practices [32]. Within this context, Carducci and colleagues have criticized media for presenting health-related information in an exaggerated method. The authors also reported that such information also affects risk perception that can result in mismanagement of the diseases [33]. In line with what is being discussed; van Bekkum and Hilton reported that this profusion of health-related information can negatively influence healthcare professionals especially at times when a crisis occurs [32]. A related example is a proposal of using hydroxychloroquine for the treatment of COVID-19. Although there is a scarcity of data about use, dosage or duration of hydroxychloroquine for prophylaxis or treatment of COVID-19 [34], the news itself was so influential that drug surge and self-medication of hydroxychloroquine was reported around the world and in Nigeria resulted in hydroxychloroquine poisoning [35]. Therefore, we urge the policymakers and regulatory authorities that as the COVID-19 related information overload the environment; the abundance of information is to be taken seriously. In addition to the dissemination of qualityassured information, myths and misinformation should be discouraged by using the same media platforms. The circulation of accurate information will prevent the population from making biased and poor decisions that will be ultimately beneficial for healthcare professionals while handling and managing the disease.

\section{Capabilities of handling and managing the COVID-19}

As health-related disasters are increasing, responses to and preparedness of the healthcare professionals and the system is the utmost challenge [36]. To meet the needs of society and to efficiently respond to the disaster, the healthcare system should be competent and equipped with the necessary provisions and supplies. Even though there are established competencies and protocols for disaster management in the literature [37], none can be developed immediately to manage a condition that erupts and spreads in little time. Additionally, as the developing world is faced with limited resources, it is important to understand the perceived capabilities of the healthcare system in crisis management. Consequently, we inquired about the competency of the nurses as well as the institute in handling and managing the COVID-19.

As expected, respondents of the study complaint of limited human resources and supplies while managing the COVID-19. The respondents were also confident that if provided with the required equipment and supplies, they are competent and equally capable to handle and manage the disease. These findings are not surprising in the context of a developing nation. The healthcare system of Pakistan faces financial limitations and out-of-pocket expenses are higher as compared to free services offered by the public institutes. Talking specifically about our study setting, the hospital carries a huge burden of patients not only from the province but also from the war-torn country of Afghanistan. It was estimated that about $40 \%$ of patients treated in Quetta are from Afghanistan [38]. Unfortunately, the Government of Baluchistan does not have a specific allocation of budget for the refugees and the resource allotment is designed only for the local population. The refugees also receive healthcare under the same budget hence the system is always faced with a shortage of medicines and other related supplies [38]. With an already resource-deprived system, it is obvious that the institute will find it difficult to handle and manage the COVID-19 in terms of human resources and supplies. Nonetheless, in addition to the foreign assistance in the shape of medical supplies and diagnostics, the central and provincial ministry is pouring heavy funds into the healthcare system that will help in managing the disease. Furthermore, the movement of people across borders is prohibited that is resulting in reducing burden on the healthcare institutes [39]. As serious efforts are observed and reported against the COVID-19, we are hopeful that the respondents' reservations are answered and the healthcare system has started assisting the professionals in handling and managing the COVID-19.

\section{Barriers in handling and managing the COVID-19}

Understanding the barriers in handling and management of diseases is an important procedure. Barriers to disease management result in high-risk behaviour and people may choose to ignore preventative or managerial measures. In addition to individualized perceptions and attitudes, cultural pressure, societal cliché and religious stigmas create barriers in handling and managing disease [40]. Our respondents also revealed some barriers that are shaping as a potential hindrance in the control and management of COVID-19 and are discussed as under.

Religious cliché and stigma have developed a certain barrier in the prevention and management of COVID-19. Especially in the developing world, we are observing practices that are resulting in avoidance of precautionary measures against the COVID-19 [41]. Where Islamic faith followers are quoting "Allah is sufficient for us; and what an excellent guardian He is." [42], drinking cow urine and hosting cow urine drinking parties to cure Corona related illness is also reported in India [43,44]. The use of sacred soil from Mount Lebanon is also practiced against COVID-19 treatment and management [45]. A simple yet effective solution to counter this stigma is by engaging the religious leaders in disseminating evidence-based information about the COVID-19. Healthcare organizations and policymakers should take the religious leaders on board as they heavily can influence the social values, practices, and beliefs of a common man with their faith-based teachings. Because of their strong influence, we believe that their role in preventing the transmission of the disease will be more proficient and acceptable as compared to the traditional healthcare role. We also agree that religion is part of the human make-up and faith healing holds a strong position in almost every community. It is high time to utilize faith in the handling and transmission of COVID-19.

Page $6 / 9$ 
Another reported barrier was COVID-19 related panic that resulted in a shortage of medical supplies and drug surges. No wonder COVID-19 is a serious issue but at the same time, the panic and fright resulted in economical and business calamity. "Panic buying" was observed and resulted in a shortage of supplies and protective materials that have shaped as a barrier in COVID-19 management. Furthermore, the price of masks, gloves, and sanitizers increased to a level that it became unaffordable for the majority of the community members. Unfortunately, the unethical and immoral practice was observed around the globe, the major affectees were people of the developing nation. Regrettably, no swift action was taken by the policymakers and there was no procedure adopted to monitor the price hike and unavailability of protective materials at the community level. In a time of crisis, such immoral practices are condemnable and place a big question mark on societal integrity and morality. We strongly urge and advise the policymakers to take swift action against the price hike and to come up with strict measures so that such practices are not repeated in the time of turmoil and crisis. To overcome the barrier at this particular moment, the availability and affordability of the materials should be strictly monitored so that people can follow evidence-based precautionary practice.

\section{Conclusion}

In a pandemic, nurses are confronted with substantial difficulties and barriers in providing healthcare services to patients. Today, the COVID-19 is an exigent catastrophe faced by the human race. In the battle against the disease, frontline nurses are playing an exceptional role in managing the disease. Findings of our study and reservations of our nurses' highlight areas of concern and scheme of improvement in the effective management of the COVID-19. Also, the results of the current study can help in planning policies and generating guidelines that can meet the healthcare needs of the society during future pandemic outbreaks of the same nature.

\section{Limitations}

Although saturation was achieved, the limited sample size is a major limitation of this study. However, with the urgency of the situation and nonavailability of COVID-19 related information, a qualitative design was the best choice to reach an in-depth investigation. Another limitation was the gender diversity as all our respondents were females. Nonetheless, being the pioneer study information retrieved from this study can lead to an expanded investigation with large and diverse sample size.

\section{Future Implications}

The findings will helpful in formulating policies and strategies for the adequate human resources as well as availability and affordability of supplies during an emergency. As the necessity of accurate information was highlighted, validity and reliability of disease-related information must be maintained especially amid a pandemic. Such efforts will effectively reduce the uncertainties faced by frontline nurses and other healthcare professionals.

\section{Abbreviations}

COVID-19: Coronavirus Disease 2019

SPH: $\quad$ Sandeman Provincial Hospital

WHO: $\quad$ World Health Organization

CDC: $\quad$ Centers for Disease Control and Prevention

UN: $\quad$ United Nations

ID: Infectious Disease

\section{Declarations}

\section{Availability of data and materials}

The datasets used and analyzed during the current study are available from the corresponding author at dr.mbashaar@gmail.com on request.

\section{Acknowledgments}

We acknowledge the nurses for their participation in the study.

\section{Funding}

No funding was received for the study.

\section{Authors' contributions}

All authors have contributed equally to the research and this manuscript. FKH and QI conceived the study and framework of the current manuscript. FUD and FS conceptualized the study design, and development of the interview guide. SH was involved in data collection, analysis, and interpretation. MB and FS conducted the literature review, drafted the preliminary manuscript, and supervised the research process. All authors read, critically revised, and approved the final manuscript. 


\section{Ethics declaration}

Institutional review board at the Faculty of Pharmacy \& Health Sciences, University of Baluchistan approved the study protocol. Written consent was taken from the respondents before the interviews. The nurses were introduced to the nature of the research prior to the beginning of the interviews, were made secure of the confidentiality of their responses and their right to withdraw from the study.

\section{Consent for publication}

This manuscript does not report personal data such as individual details, images, or videos. Therefore, consent for publication is not applicable.

\section{Competing interests}

The authors declare that they have no competing interests.

\section{References}

1. Lam SK, Kwong EW, Hung MS, Pang SM, Chiang VC: Nurses' preparedness for infectious disease outbreaks: A literature review and narrative synthesis of qualitative evidence. J Clinc Nurs. 2018;27(7-8):e1244-e1255.

2. Infectious diseases [https://www.who.int/topics/infectious_diseases/en/]

3. Petersen E, Petrosillo N, Koopmans M, Beeching N, Di Caro A, Gkrania-Klotsas E, Kantele A, Kohlmann R, Lim P-L, Markotic A: Emerging infections-an increasingly important topic: review by the Emerging Infections Task Force. Clin Microbiol Infect. 2018;24(4):369-375.

4. Jones KE, Patel NG, Levy MA, Storeygard A, Balk D, Gittleman JL, Daszak P: Global trends in emerging infectious diseases. Nature. 2008;451(7181):990993.

5. Coronavirus Disease 2019 (COVID-19): Situation Summary [https://www.cdc.gov/coronavirus/2019-ncov/summary.html]

6. Coronavirus disease (COVID-19) outbreak [https://experience.arcgis.com/experience/685d0ace521648f8a5beeeee1b9125cd]

7. Report of the WHO-China Joint Mission on Coronavirus Disease 2019 (COVID-19) [https://www.who.int/docs/default-source/coronaviruse/who-chinajoint-mission-on-covid-19-final-report.pdf]

8. Nyblade L, Stockton MA, Giger K, Bond V, Ekstrand ML, Mc Lean R, Mitchell EM, La Ron EN, Sapag JC, Siraprapasiri T: Stigma in health facilities: why it matters and how we can change it. BMC Medicine. 2019;17(1):25.

9. Zarei N, Joulaei H, Darabi E, Fararouei M: Stigmatized attitude of healthcare providers: a barrier for delivering health services to HIV positive patients. Int J Community Based Nurs Midwifery. 2015;3(4):292-300.

10. Kohrt BA, Jordans MJ, Turner EL, Sikkema KJ, Luitel NP, Rai S, Singla DR, Lamichhane J, Lund C, Patel V: Reducing stigma among healthcare providers to improve mental health services (RESHAPE): protocol for a pilot cluster randomized controlled trial of a stigma reduction intervention for training primary healthcare workers in Nepal. Pilot Feasibility Stud. 2018;4(1):36.

11. Kieft RA, de Brouwer BB, Francke AL, Delnoij DM: How nurses and their work environment affect patient experiences of the quality of care: a qualitative study. BMC Health Serv Res. 2014;14(1):249.

12. Hughes R: Patient safety and quality: An evidence-based handbook for nurses, vol. 3: Agency for Healthcare Research and Quality Rockville, MD; 2008.

13. Lam KK, Hung SYM: Perceptions of emergency nurses during the human swine influenza outbreak: A qualitative study. Int Emerg Nurs. 2013;21(4):240246.

14. Shih FJ, Turale S, Lin YS, Gau ML, Kao CC, Yang CY, Liao YC: Surviving a life-threatening crisis: Taiwan's nurse leaders' reflections and difficulties fighting the SARS epidemic. J Clinc Nurs. 2009;18(24):3391-3400.

15. Kitzinger J: Qualitative research: introducing focus groups. Brit Med J. 1995;311(7000):299-302.

16. Berg BL, Lune H, Lune H: Qualitative research methods for the social sciences, vol. 5: Pearson Boston, MA; 2004.

17. Krueger RA: Focus groups: A practical guide for applied research: Sage. Inc; 2009.

18. Stewart DW, Shamdasani PN: Focus groups: Theory and practice, vol. 20: Sage Publications; 2014.

19. Mullen PD, Reynolds R: The potential of grounded theory for health education research: linking theory and practice. Health Educ Monogr. 1978;6(3):280294.

20. Entwistle VA, Renfrew MJ, Yearley S, Forrester J, Lamont T: Lay perspectives: advantages for health research. Brit Med J. 1998;316(7129):463-466.

21. Shahzad F, Saleem F, Iqbal Q, Haque N, Haider S, Salman M, Masood I, Hassali MA, Iftikhar S, Bashaar M: A cross-sectional assessment of health literacy among hypertensive community of Quetta City, Pakistan. Biomed J Sci Tech Res. 2018;4:1-9.

22. Brace-Govan J: Issues in snowball sampling: The lawyer, the model and ethics. Qual Res J. 2004;4(1):52.

23. Patton MQ: Qualitative research \& evaluation methods: Integrating theory and practice: Sage publications; 2014.

24. Wild D, Grove A, Martin M, Eremenco S, McElroy S, Verjee-Lorenz A, Erikson P: Principles of good practice for the translation and cultural adaptation process for patient-reported outcomes (PRO) measures: report of the ISPOR Task Force for Translation and Cultural Adaptation. Value Health.

2005;8(2):94-104.

25. Guest G, MacQueen KM, Namey EE: Introduction to applied thematic analysis. Applied Thematic Analysis 2012, 3:20.

26. National action plan for corona virus disease (COVID-19) Pakistan [https://www.nih.org.pk/wp-content/uploads/2020/03/COVID-19-NAP-V2-13-March2020.pdf?_cf_chl_jschl_tk_=4cb728a33fffac46ccc6566a34eb292a317f4f74-1584542039-0- 
27. Shortage of protective equipment hampers efforts against coronavirus [https://www.dawn.com/news/1533533]

28. About Balochistan [http://balochistan.gov.pk/explore-balochistan/about-balochistan/]

29. Coronavirus disease (COVID-2019) situation reports [https://www.who.int/emergencies/diseases/novel-coronavirus-2019/situation-reports]

30. Bavier A: An overview of health challenges faced by nurses. J Appl Behav Res. 2018;23(1):e12118.

31. Gatchel RJ: The importance of the profession of nursing in health care: Current and future issues. J Appl Behav Res. 2018;23(1):e12127.

32. van Bekkum JE, Hilton S: Primary care nurses' experiences of how the mass media influence frontline healthcare in the UK. BMC Fam Prac. 2013;14(1):178.

33. Carducci A, Alfani S, Sassi M, Cinini A, Calamusa A: Mass media health information: quantitative and qualitative analysis of daily press coverage and its relation with public perceptions. Patient Educ Couns. 2011;82(3):475-478.

34. Information for clinicians on therapeutic options for COVID-19 patients [https://www.cdc.gov/coronavirus/2019-ncov/hcp/therapeutic-options.html]

35. Nigeria reports poisonings from possible coronavirus drug chloroquine [https://nypost.com/2020/03/22/nigeria-reports-poisonings-from-possiblecoronavirus-drug-chloroquine/]

36. Loke AY, Fung OWM: Nurses' competencies in disaster nursing: Implications for curriculum development and public health. Int J Environ Res Public Health. 2014;11(3):3289-3303.

37. Daily E, Padjen P, Birnbaum M: A review of competencies developed for disaster healthcare providers: limitations of current processes and applicability. Prehosp Disaster Med. 2010;25(5):387-395.

38. Healthcare in Balochistan under stress from Afghan patients [https://dailytimes.com.pk/58260/healthcare-in-balochistan-under-stress-from-afghanpatients/]

39. Pakistan shuts border with Iran, Afghanistan over virus [https://www.aa.com.tr/en/asia-pacific/pakistan-shuts-border-with-iran-afghanistan-overvirus/1767278]

40. Mental health: Culture, race, and ethnicity-A supplement to mental health: A report of the surgeon general [https://drum.lib.umd.edu/handle/1903/22834]

41. Coronavirus disease (COVID-19) advice for the public [https://www.who.int/emergencies/diseases/novel-coronavirus-2019/advice-for-public]

42. The Noble Qur'an: Surah Ali 'Imran 3:173: Pro Bono Project; 2016.

43. Coronavirus: Is drinking cow urine any help? [https://www.bbc.com/news/world-asia-india-51910099]

44. Coronavirus: Hindu group hosts 'cow urine drinking party' to cure illness despite lack of evidence [https://www.independent.co.uk/news/world/asia/coronavirus-news-hindu-cow-urine-drinking-party-india-a9402491.html]

45. Sacred soil, cow urine and other riruals: people turn to religion to deal with the corona virus [https://www.albawaba.com/node/sacred-soil-cow-urine-andother-rituals-people-turn-religion-deal-coronavirus-1345263] 